\section{A self-care with Ou MC decrescendo phenomenon may possibly prevent mild COVID-19 disease developing into severe or critical stage}

\author{
Ming Cheh Ou, ${ }^{1}$ Dennis Ou, ${ }^{2}$ \\ Chung Chu Pang ${ }^{3}$
}

${ }^{1}$ Department of Obstetrics and Gynecology, Zhong-Xiao Branch, Taipei City Hospital, Taipei City, Taiwan; ${ }^{2}$ Department of Mechanical Engineering and Biomedical Engineering, Carnegie Mellon University, Pittsburgh, USA; ${ }^{3}$ Department of Occupational Therapy, National Taiwan University, Taipei City, Taiwan

\begin{abstract}
$\mathrm{Ou}$ MC decrescendo phenomenon treatment $(\mathrm{OuDPt})$ is a self-administered practice in which the interactions of the anatomical axes result in a physiological response and produce a zone under the contralateral hand with decreased pain or inflammation. OuDPt has shown an effect to alleviate cough, rhinorrhea, headache, acute edematous swellings, dysmenorrhea, endometriosis and joint pain that are related to inflammatory reaction. Most symptoms with coronavirus infection disease 2019 (COVID-19) as fever, cough, myalgia or diarrhea are also associated with inflammation. If the inflammation by severe acute respiratory syndrome coronavirus 2 is suppressed, mild COVID-19 disease may not develop into severe or critical stage. Further investigations are warranted.
\end{abstract}

\section{Introduction}

Ou MC decrescendo phenomenon treatment (OuDPt) is a self-therapy practice in which the interactions of the anatomical axes result in a physiological response and produce a zone under the hand with decreased pain or inflammation. ${ }^{1,2}$

OuDPt may improve degenerative diseases, organ dysfunction and malignant change, which indicates the normalization of tissue function. ${ }^{3}$ Gradual decline of tissue function is associated with the development of physiological characteristics of aging and can make people susceptible to various dis- eases. ${ }^{4}$ Thus, restoration of tissue function of aged people may prevent the diseases developing into severe or critical stage, though restoration of tissue function alone may not be sufficient to suppress or cure diseases.

\section{Anti-inflammation by Ou MC decrescendo phenomenon treatment}

Restoration of normal tissue function may re-establish host defense systems, which will contribute to defense against microorganisms, inflammation, degenerative changes, and tumors. OuDPt has shown an effect to alleviate rhinorrhea, headache, acute edematous swelling, supine cough, dysmenorrhea, endometriosis, joint pain and also causes recovery of organ dysfunction or neoplastic disease..$^{3,5-7}$ Studies show senescent cells with decline function contribute pro-inflammatory factors; thus, restoration of tissue function of senescent cells function may suppress such proinflammatory process. ${ }^{8}$ OuDPt is more effective with the contralateral hand than the ipsilateral hand in producing the OuDP. This finding implies that the axes of embryonic polarity, especially the left-right axis, form the potential mechanism underlying the OuDP. 7,9

\section{Inflammation of COVID-19 diseases}

Severe acute respiratory syndrome coronavirus 2 (SARS-CoV-2) may impair protective immune response, and massive destruction of the affected tissues will occur, especially in organs that have high ACE2 expression, such as intestine and kidney. The damaged cells induce innate inflammation in the lungs that is largely mediated by pro-inflammatory macrophages and granulocytes. ${ }^{10}$ Thus, most symptoms with coronavirus infection disease 2019 (COVID-19) as fever, cough, myalgia or diarrhea are associated with inflammation. If the inflammation by SARS-CoV-2 virus is suppressed, mild COVID-19 disease may not develop into severe or critical stage. OuDPt shows a capability to suppress inflammation, , $5-7$ which may possibly suppress inflammations by COVID-19 disease. However, normalization of tissue function may not always be sufficient to resolve diseases.
Correspondence: Ming Cheh Ou, Department of Obstetrics \& Gynecology of Taipei City Hospital, Taipei city, Taiwan.

Tel.: 886.979308190.

E-mail: peitouwan@gmail.com

Key words: COVID-19; anti-inflammation; Ou MC decrescendo phenomenon; tissue function normalization; elderly.

Acknowledgments: the authors thank the help by Miss Ou, Yi Jen.

Conflict of interests: the authors declare no conflict of interests.

Availability of data and materials: The data underlying this article are within the article and its supplementary materials.

Ethics approval and consent to participate: Ethics approval with No.82075656 document, Department of Health, Executive Yuan, ROC (Taiwan). All patients provided consent for participation.

Received for publication: 7 June 2020.

Revision received: 14 July 2020.

Accepted for publication: 14 October 2020.

This work is licensed under a Creative Commons Attribution-NonCommercial 4.0 International License (CC BY-NC 4.0).

${ }^{\circ}$ Copyright: the Author(s), 2020

Licensee PAGEPress, Italy

Geriatric Care 2020; 6:9171

doi:10.4081/gc.2020.9171

\section{Ou MC decrescendo phenomenon treatment for inflammation}

For COVID-19 disease is a systemic infectious disease, OuDPt with all 3 anatomical axes is recommended. Figure 1 and Table 1 demonstrate the performance of OuDpt.

The effectiveness OuDP can be increased by decreasing the distance between the hand and the lesion, increasing treatment duration, increasing the treatment frequency and individualized by patients. Nonetheless, the appropriate duration and frequency of OuDPt application for the treatment of different diseases requires further study.

\section{Limitations}

Though OuDPt appears to be consistently effective for treating a wide variety 


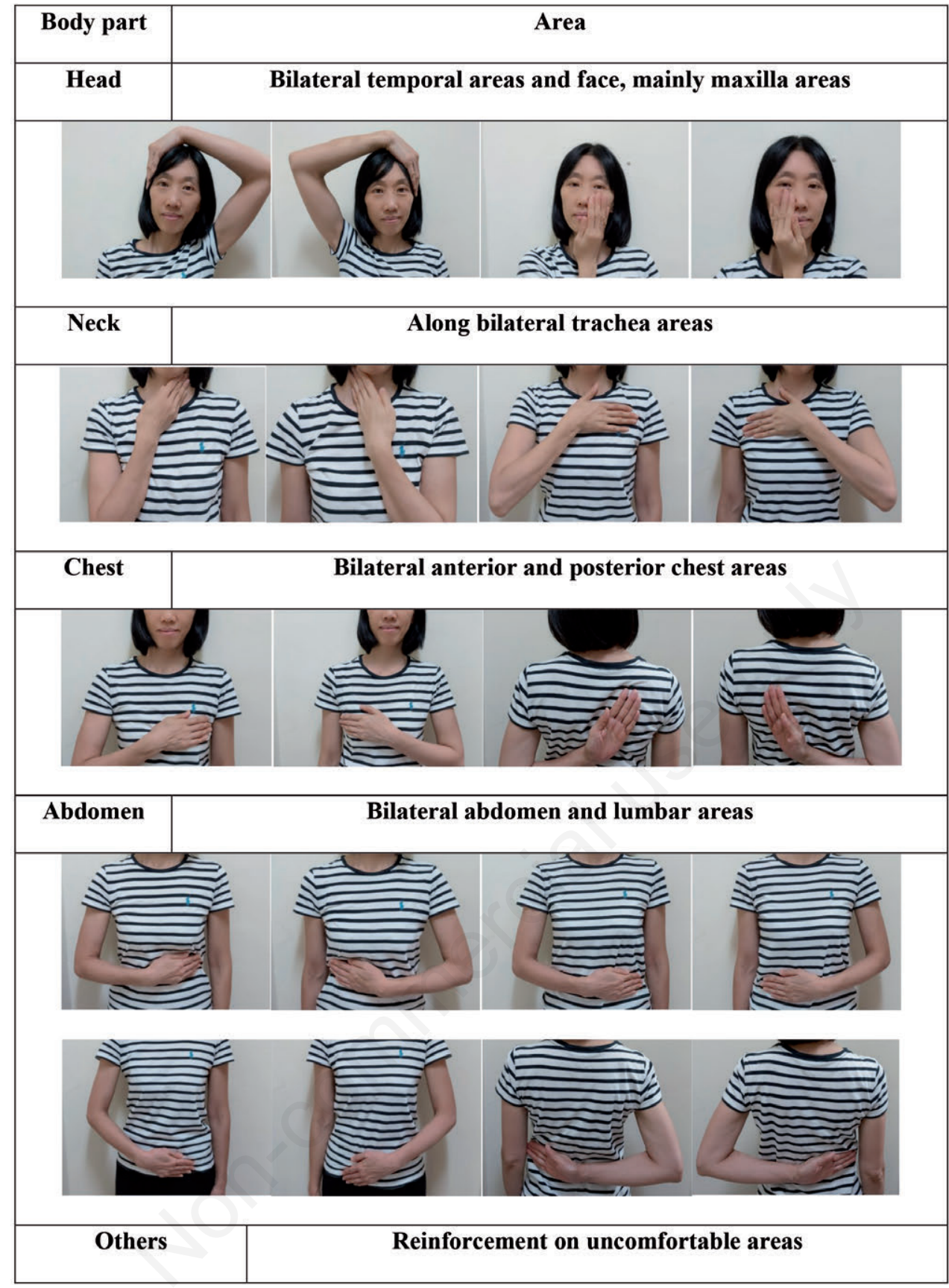

Figure 1. Areas to perform Ou decrescendo phenomenon treatment for inflammation. Five seconds or more on each site, reinforcement on uncomfortable areas, especially chest, maxilla and trachea areas for COVID-19 disease ${ }^{1,5}$ (photos demonstrated by Pang CC, author).

Table 1. Practical points for Ou decrescendo phenomenon treatment (OuDPt).

1. The OuDPt is mainly performed by the patient by placing the contralateral hand directly on the affected area via the left-right, dorsoventral or vertical axes. Application along more axes has shown to be more effective

2. For lesions on or adjoining the midline of the body, OuDPt is applied first to one side of the lesion and then the other, using the contralateral hand

3. The effects of OuDPt are related to the duration and frequency of administration and the distance between the hand and the lesion

4. If OuDPt is not efficacious, measures such as getting the hand nearer to the lesion and increasing the duration or frequency of administration may be helpful. Longer duration may cause organ compression and pressing too forcefully may cause tumor bleeding or exfoliation

5. OuDPt may contaminate an open wound

6. Different positioning may be useful when performing OuDPt

7. Severe emotional disturbance may possibly affect effectiveness 
of diseases, normalization of tissue function may not always be sufficient to resolve diseases. OuDPt is effective in treating pain induced by dysfunctional tissue such as that of degenerative joint disease or endometriosis, but may not be sufficient to treat a disease unrelated to tissue dysfunction. For infectious diseases, OuDPt does not resolve the infections but may alleviate inflammations which aggravate the diseases. . $^{3,9,11}$

\section{Conclusions}

Physiologic and functional status varies among people. This wide heterogeneity means both preventive and therapeutic interventions should be considered for preserving function and maximizing quality of life. OuDPt shows to provide immediate and long-term improvement for various diseases and can be performed by people themselves and is easy to carry out with little cost (Figure 1 and Table 1). It indicates that OuDPt can be availed for preventing diseases from developing into severe or critical stage but also a way to keep people healthy as a prevention of diseases, espe- cially for the elderly. Further studies are warranted.

\section{References}

1. Ou MC, Pang CC, Ou D, Su CH. The implications of abdominal palpation with $\mathrm{Ou} \mathrm{MC}$ manipulation for women with acute abdomen. Am J Emerg Med 2012;30:421-5.

2. Ou MC, Ou D, Pang CC. The relation between human bilateral symmetry and disease. Proc Physiol Soc 2014;31: PCA132.

3. Ou MC, Ou D, Pang CC. A primitive approach to $\mathrm{Ou} \mathrm{MC}$ decrescendo phenomenon with a hands-on therapy - The relation between human bilateral symmetry and disease. Nat Sci 2014;6:88-98.

4. McHugh D, Gil J. Senescence and aging: causes, consequences, and therapeutic avenues. J Cell Biol 2018;217: 65-77.

5. Ou MC, Ou D, Pang CC. The antiinflammatory effect by interactions of human body anatomical axes for dysmenorrhea caused by endometriosis. J Assist Reprod Genet 2018;35:2097.

6. $\mathrm{Ou} \mathrm{MC}, \mathrm{Ou}$, Pang CC.
Dysmenorrhea relieved by the interactions of human body anatomical axes indicate an anti-inflammatory effect by normalization of tissue function. Proc Physiol Soc 2019;43:PC247.

7. Lasry A, Ben-Neriah Y. Senescenceassociated inflammatory responses: aging and cancer perspectives. Trends Immunol 2015;36:217-28.

8. Ou MC, Ou D, Pang C. The possible role of embryonic polarity axes for the normalization of tissue function induced by the interaction between human bilateral parts. Integr Cancer Sci Therap 2015;3:329-35.

9. Ou MC, Pang CC, Ou D. The Implication of tumor regression by a three-dimensional human body polarity system formed with human body anatomical axes. Taiwan J Obstet Gynecol 2017;56:116-9.

10. Tay MZ, Poh CM, Rénia L, et al. The trinity of COVID-19: immunity, inflammation and intervention. Nat Rev Immunol 2020;20:363-74.

11. Ou MC, Pang CC, Ou D. The implication of cancer regression by the interactions with human anatomical axes - an active prevention for cancer. Cancer Arc 2019;1:1-3. 